Volume 24 (2018) 18-30

DOI: $10.24330 /$ ieja.440141

\title{
SUPPLEMENTS IN COATOMIC MODULES HAVING THE COMPLETE MAX-PROPERTY
}

\author{
Mame Demba Cissé, Lamine Ngom, Djiby Sow and Rachid Tribak \\ Received: 30 August 2017; Revised: 16 December 2017; Accepted: 20 December 2017 \\ Communicated by Christian Lomp \\ Dedicated to the memory of Professor John Clark
}

\begin{abstract}
Let $R$ be a ring with identity. A right $R$-module $M$ has the complete max-property if the maximal submodules of $M$ are completely coindependent (i.e., every maximal submodule of $M$ does not contain the intersection of the other maximal submodules of $M$ ). A right $R$-module is said to be a good module provided every proper submodule of $M$ containing $\operatorname{Rad}(M)$ is an intersection of maximal submodules of $M$. We obtain a new characterization of good modules. Also, we study good modules which have the complete maxproperty. The second part of this paper is devoted to investigate supplements in a coatomic module which has the complete max-property.
\end{abstract}

Mathematics Subject Classification (2010): 16D10, 16D99

Keywords: Coatomic module, completely coindependent, complete maxproperty, good module, maximal submodule, supplement submodule

\section{Introduction}

Let $R$ be a unitary ring and $M$ a right $R$-module. A submodule $N$ of $M$ is called small in $M$ (written $N \ll M$ ) if for every proper submodule $L$ of $M, N+L \neq M$. A submodule $L$ of $M$ is called coclosed in $M$ if $L / K$ is not small in $M / K$ for any proper submodule $K$ of $L$. We denote by $\operatorname{Rad}(M)$ the radical of $M$. A module $M$ is called coatomic if every proper submodule of $M$ is contained in a maximal submodule, that is, $\operatorname{Rad}(M / N) \neq 0$ for every proper submodule $N \leq M$. Let $L$ be a submodule of $M$. A submodule $K$ of $M$ is called a supplement of $L$ in $M$ if $K$ is minimal with respect to the property $M=L+K$; equivalently, $M=L+K$ and $K \cap L \ll K$. A submodule $P$ of $M$ is called a supplement submodule if $P$ is a supplement of some submodule of $M$. The module $M$ is called supplemented if every submodule of $M$ has a supplement in $M$. A module $M$ is called semilocal if $M / \operatorname{Rad}(M)$ is semisimple. A module $M$ is called cosemisimple (or a $V$-module) if every simple $R$-module is $M$-injective, or equivalently, every proper submodule of $M$ is an intersection of maximal submodules (see [7, 23.1]). A module $M$ is called a 
good module if $M / \operatorname{Rad}(M)$ is a cosemisimple module (see [7, 23.3]). A non-empty family of submodules $N_{i}(i \in I)$ of a module $M$ is called coindependent if, for any $j \in I$ and any finite subset $J$ of $I \backslash\{j\}, N_{j}+\bigcap_{i \in J} N_{i}=M$. The family $N_{i}(i \in I)$ is called completely coindependent if, for every $j \in I, N_{j}+\bigcap_{i \neq j} N_{i}=M$ (see [4, p. 8]). Following [6, p. 74], a module $M$ is said to have the complete max-property if the maximal submodules of $M$ form a completely coindependent set of submodules of $M$. In this paper, we adopt the convention that the intersection of an empty set of submodules of a module $M$ is $M$ itself.

In Section 2, we provide some new characterizations of good modules (Theorem 2.3). Also, we investigate the interplay between the complete max-property and each one of the properties coatomic and good.

The investigations in Section 3 focus on supplements in a coatomic module which has the complete max-property. After characterizing them, we show that for a coatomic module $M$, if $M$ has the complete max-property, then any supplement submodule in $M$ has also the complete max-property. In addition, we prove that if $M$ is a coatomic module which has the complete max-property and $F$ is a supplement of a submodule $K$ in $M$, then $\Delta_{F}(M)=K+\operatorname{Rad}(F)=K+\operatorname{Rad}(M)$ where $\Delta_{F}(M)$ denotes the intersection of the maximal submodules of $M$ not containing $F$.

Throughout this paper, $R$ will denote an associative ring with identity and all modules are unitary right $R$-modules. By $\mathbb{Q}$ and $\mathbb{Z}$ we denote the ring of rational and integer numbers, respectively.

\section{Good modules having the complete max-property}

Recall that a module $M$ is said to be a good module if for any module $N$ and any homomorphism $f: M \rightarrow N, f(\operatorname{Rad}(M))=\operatorname{Rad}(f(M))$. In this section, we obtain a new characterization of good modules. Moreover, we shed some light on good modules which have the complete max-property.

Let $F$ be a submodule of a module $M$. We follow the notation of [3]. So the intersection of all maximal submodules of $M$ containing $F$ will be denoted by $\operatorname{Rad}_{F}(M)$. It is easily seen that $F+\operatorname{Rad} M \subseteq \operatorname{Rad}_{F}(M)$. On the other hand, we do not have equality, in general, as shown in [3, Remark 3.4]. In the same vein, we exhibit the following examples.

Example 2.1. (i) Consider the submodule $F=p^{k} \mathbb{Z}$ of $M=\mathbb{Z}$ for some prime integer $p$ and some integer $k \geq 2$. We have $\operatorname{Rad}(M)=0$. So $F+\operatorname{Rad}(M)=F$, but $\operatorname{Rad}_{F}(M)=p \mathbb{Z}$. 
(ii) Let $p$ and $q$ be two prime integers such that $p \neq q$. Consider the submodule $F=p^{n} q^{m} \mathbb{Z}$ of $M=\mathbb{Z}$, where $n$ and $m$ are natural numbers with $n \geq 2$ and $m \geq 2$. Clearly, $\operatorname{Rad}(M)=0$. Then $F+\operatorname{Rad}(M)=F$. However, $\operatorname{Rad}_{F}(M)=p q \mathbb{Z}$.

In [3], the authors provided some conditions under which $\operatorname{Rad}_{F}(M)=F+\operatorname{Rad} M$ for a submodule $F$ of $M$. Among other results, it is shown in [3, Proposition 3.8] that if $M$ is a good module, then $\operatorname{Rad}_{F}(M)=F+\operatorname{Rad} M$ for any submodule $F$ of $M$. The next proposition shows that the converse of this result is true.

Proposition 2.2. The following statements are equivalent for a module $M$ :

(i) $M$ is a good module;

(ii) Every proper submodule of $M$ containing $\operatorname{Rad}(M)$ is an intersection of maximal submodules of $M$;

(iii) $\operatorname{Rad}_{F}(M)=F+\operatorname{Rad}(M)$ for every submodule $F$ of $M$.

Proof. (i) $\Leftrightarrow$ (ii) This follows from [7, 23.1 and 23.3].

(i) $\Rightarrow$ (iii) By [3, Proposition 3.8].

(iii) $\Rightarrow$ (ii) Let $L$ be a proper submodule of $M$ such that $\operatorname{Rad}(M) \subseteq L$. By hypothesis, we have $\operatorname{Rad}_{L}(M)=L+\operatorname{Rad}(M)=L$. Hence $L$ is an intersection of maximal submodules of $M$.

Let $F$ be a submodule of a module $M$. The intersection of the maximal submodules of $M$ not containing $F$ will be denoted by $\Delta_{F}(M)$.

Theorem 2.3. The following statements are equivalent for a module $M$ :

(i) $M$ is a good module;

(ii) $\operatorname{Rad}_{F}(M)=F+\operatorname{Rad}(M)$ for every submodule $F$ of $M$;

(iii) $\operatorname{Rad}_{F}(M) \subseteq F+\Delta_{F}(M)$ for every submodule $F$ of $M$;

(iv) For any submodule $F$ of $M$ and any collection of maximal submodules $N_{i}$ $(i \in I)$ of $M$, we have $F+\left(\bigcap_{i \in I} N_{i}\right)=M$ or $F+\left(\bigcap_{i \in I} N_{i}\right)$ is an intersection of maximal submodules of $M$;

(v) For any submodule $F$ of $M$, we have $F+\Delta_{F}(M)=M$ or $F+\Delta_{F}(M)$ is an intersection of maximal submodules of $M$.

Proof. (i) $\Leftrightarrow$ (ii) This follows from Proposition 2.2.

(ii) $\Leftrightarrow$ (iii) By [3, Proposition 3.5].

(i) $\Rightarrow$ (iv) This follows from Proposition 2.2.

(iv) $\Rightarrow(\mathrm{v}) \Rightarrow$ (iii) These are obvious. 
Remark 2.4. From Theorem 2.3, it follows that a module $M$ for which

$$
F+\Delta_{F}(M)=M \text { for all } F \leq M
$$

is a good module.

Definition 2.5. A module $M$ is said to have the strong max-property if for every submodule $F$ of $M$, we have $F+\Delta_{F}(M)=M$.

We shall say that a module $M$ has the max-property if the maximal submodules of $M$ form a coindependent set of submodules of $M$ (i.e., $M=L+\cap_{i=1}^{n} L_{i}$ for every positive integer $n$ and distinct maximal submodules $L, L_{i}(1 \leq i \leq n)$ of $M$ ) (see $[6])$.

It is clear that the following implications hold:

Strong max-property $\Rightarrow$ complete max-property $\Rightarrow$ max-property.

The following lemma is a direct consequence of [6, Proposition 4.2 and Theorem $6.8]$.

Lemma 2.6. Let $M$ be an $R$-module which has the complete max-property such that $M / \operatorname{Rad}(M)$ is coatomic. Then $M$ is a semilocal module.

Proposition 2.7. Any module which has the strong max-property is semilocal.

Proof. Let $M$ be a module with the strong max-property. By Theorem 2.3, $M$ is a good module. Thus $M / \operatorname{Rad}(M)$ is a cosemisimple module. Hence $M / \operatorname{Rad}(M)$ is a coatomic module. Note that $M$ has the complete max-property. Applying Lemma 2.6 , we conclude that $M$ is semilocal.

Theorem 2.8. The following statements are equivalent for a module $M$ :

(i) $M$ is a good module and $M$ has the complete max-property;

(ii) $M$ has the strong max-property.

Proof. (i) $\Rightarrow$ (ii) Suppose that $F+\Delta_{F}(M) \neq M$ for some submodule $F$ of $M$. Then $F+\Delta_{F}(M)$ is an intersection of maximal submodules of $M$ by Theorem 2.3. Therefore $\operatorname{Rad}_{F}(M) \subseteq F+\Delta_{F}(M)$ and hence $\operatorname{Rad}_{F}(M)+\Delta_{F}(M)=F+\Delta_{F}(M)$. $\operatorname{But}_{\operatorname{Rad}_{F}}(M)+\Delta_{F}(M)=M$ by [6, Proposition 6.1]. So $F+\Delta_{F}(M)=M$, a contradiction. This shows that $M$ has the strong max-property.

(ii) $\Rightarrow$ (i) This is immediate.

In the next example we present a coatomic good module which is not semilocal.

Example 2.9. Let $R$ be a right cosemisimple ring (i.e., $R$ is a right $V$-ring) which is not semisimple (e.g., we take a field $F$ and $R=\prod_{i \geq 1} F_{i}$ where $F_{i}=F$ for all $i \geq 1)$. Then the $R$-module $R_{R}$ is coatomic, but $R_{R}$ is not semilocal since $\operatorname{Rad}\left(R_{R}\right)=0$. Moreover, it is clear that $R_{R}$ is a good module. 
From Lemma 2.6, we get the following proposition which provides a sufficient condition for a coatomic module to be semilocal.

Proposition 2.10. Let $M$ be a coatomic module which has the complete maxproperty. Then $M$ is semilocal. In particular, $M$ is a good module.

Combining Theorem 2.8 and Proposition 2.10, we obtain the following result.

Corollary 2.11. Let $M$ be a coatomic module. Then the following statements are equivalent:

(i) $M$ has the complete max-property;

(ii) $M$ has the strong max-property.

The next example shows that, in general, a good module need not be coatomic.

Example 2.12. (i) Let $p$ be a prime integer and consider the $\mathbb{Z}$-module $M=$

$\oplus_{n \geq 1} \mathbb{Z} / p^{n} \mathbb{Z}$. Since $\frac{\mathbb{Z} / p^{n} \mathbb{Z}}{\operatorname{Rad}\left(\mathbb{Z} / p^{n} \mathbb{Z}\right)}$ is a semisimple module for all $n \geq 1, \mathbb{Z} / p^{n} \mathbb{Z}$ is a good module for all $n \geq 1$. Thus $M$ is a good module by [7, 23.4]. However, $M$ is not coatomic by [8, Lemma 1.2].

(ii) Let $M$ be a module such that $\operatorname{Rad}(M)=M$. Then $M$ is a good module as $M / \operatorname{Rad}(M)=0$ is semisimple. On the other hand, $M$ is not coatomic.

In the next example, we exhibit a coatomic module which is not a good module.

Example 2.13. Let $R$ be a ring which is not a right $V$-ring such that $\operatorname{Rad}(R)=0$ (e.g., we can take $R=\mathbb{Z}$ ). Clearly, the $R$-module $M=R_{R}$ is coatomic, but $M$ is not a good module.

Note that the class of semilocal modules is a proper subclass of the class of good modules (see Example 2.9). From [4, 2.8(8)], it follows that any semilocal module with a small radical is coatomic. This result can be extended to good modules as shown below.

Proposition 2.14. Let $M$ be a good module with a small radical. Then $M$ is coatomic.

Proof. Let $N$ be a proper submodule of $M$. Then $N+\operatorname{Rad}(M) \neq M$ as $\operatorname{Rad}(M) \ll$ $M$. Since $M$ is a good module, $N+\operatorname{Rad}(M)$ is an intersection of maximal submodules of $M$. The result follows.

\section{Applications to supplement submodules}

Our goal in this section is to characterize supplement submodules in a coatomic module which has the complete max-property. We begin with the following result on coclosed submodules of a coatomic good module. 
Proposition 3.1. Let $M$ be a coatomic good module and let $F$ be a submodule of $M$ such that $\operatorname{Rad}(M) \subseteq F$. Then the following assertions are equivalent:

(i) $F$ is coclosed in $M$;

(ii) $F$ is coatomic and $\operatorname{Rad}(F)=\operatorname{Rad}(M)$.

Proof. (i) $\Rightarrow$ (ii) From [2, Lemma 4.1], it follows that $F$ is coatomic. Moreover, we have $\operatorname{Rad}(F)=F \cap \operatorname{Rad}(M)$ by [4, 3.7]. As $\operatorname{Rad}(M) \subseteq F$, we obtain $\operatorname{Rad}(F)=$ $\operatorname{Rad}(M)$.

(ii) $\Rightarrow$ (i) Let $L \leq F$ such that $F / L \ll M / L$. Then $F / L \subseteq \operatorname{Rad}(M / L)$. Since $M$ is a good module, we have

$$
\operatorname{Rad}(M / L)=(L+\operatorname{Rad}(M)) / L=(L+\operatorname{Rad}(F)) / L .
$$

Therefore $\operatorname{Rad}(M / L) \subseteq \operatorname{Rad}(F / L)$ by $[4,2.8$ (1)]. So $F / L \subseteq \operatorname{Rad}(F / L)$. Hence, $F / L=\operatorname{Rad}(F / L)$. As $F$ is coatomic, it follows that $F / L=0$; that is, $L=F$. This completes the proof.

It was shown in [5, Theorem 2.1] that if $F$ is a supplement of a submodule $K$ in a module $M$, then it is possible to define a bijective map between maximal submodules of $F$ and maximal submodules of $M$ which contain $K$. In the next result, we use this fact to characterize supplement submodules in a coatomic module.

Proposition 3.2. Let $F$ and $K$ be submodules of a coatomic module $M$. Then the following statements are equivalent:

(i) $F$ is a supplement of $K$ in $M$;

(ii) (1) $F$ is coatomic, and

(2) for any submodule $N$ of $F, N$ is a maximal submodule of $F$ if and only if $N=F \cap L$ for some maximal submodule $L$ of $M$ with $K \subseteq L$.

Proof. (i) $\Rightarrow$ (ii) This follows from [2, Lemma 4.1] and [5, Theorem 2.1].

(ii) $\Rightarrow$ (i) Suppose that $K+F \neq M$. Since $M$ is coatomic, there exists a maximal submodule $X$ of $M$ such that $K+F \subseteq X$. By (2), $F \cap X=F$ is a maximal submodule of $F$, a contradiction. So $K+F=M$. Now let $H$ be a proper submodule of $F$. Since $F$ is coatomic, $H \subseteq Y$ for some maximal submodule $Y$ of $F$. By hypothesis, there exists a maximal submodule $Z$ of $M$ such that $K \subseteq Z$ and $Y=F \cap Z$. Therefore $H+K \subseteq Y+K=(F \cap Z)+K \subseteq Z$. It follows that $H+K \neq M$. This proves that $F$ is a supplement of $K$ in $M$.

Theorem 3.3. Let $M$ be a coatomic module which has the complete max-property. Then the following statements about a submodule $F$ of $M$ are equivalent:

(i) $F$ is a supplement in $M$; 
(ii) $F$ is coatomic and $F \cap \operatorname{Rad}(M)=\operatorname{Rad}(F)$;

(iii) $F \cap \operatorname{Rad}(M) \ll F$;

(iv) $F$ is coclosed in $M$;

(v) $F$ is a supplement of $\Delta_{F}(M)$ in $M$;

(vi) $F$ is a supplement of $\operatorname{Rad}(M)$ in $\operatorname{Rad}_{F}(M)$;

(vii) $F \cap \Delta_{F}(M) \ll F$;

(viii) $F$ is coatomic and $F \cap \Delta_{F}(M)=\operatorname{Rad}(F)$.

Proof. Note that $M$ is a good module by Proposition 2.10. Applying Theorems 2.3 and 2.8, we conclude that $\operatorname{Rad}_{N}(M)=N+\operatorname{Rad}(M)$ and $N+\Delta_{N}(M)=M$ for every submodule $N$ of $M$.

(i) $\Rightarrow$ (v) Assume that $F$ is a supplement of a submodule $U$ in $M$. Note that $\operatorname{Rad} M \ll M$ as $M$ is coatomic. So $F$ is also a supplement of $U+\operatorname{Rad} M$ in $M$ by [4, 20.4 (4)]. Since $\operatorname{Rad}_{U}(M)=U+\operatorname{Rad}(M), F$ is a supplement of $\operatorname{Rad}_{U}(M)$ in $M$. Moreover, we have $\Delta_{F}(M) \subseteq \operatorname{Rad}_{U}(M)$ as $F+U=M$. Since $F+\Delta_{F}(M)=M$, it follows that $F$ is a supplement of $\Delta_{F}(M)$ in $M$ by [4, 20.4 (1)].

(v) $\Rightarrow$ (vii) This is obvious.

(vii) $\Rightarrow$ (iv) Assume that $\Delta_{F}(M) \cap F \ll F$. Since $F+\Delta_{F}(M)=M$, it follows that $F$ is a supplement of $\Delta_{F}(M)$ in $M$. Hence $F$ is coclosed in $M$ by [4, 20.2].

(iv) $\Rightarrow$ (ii) From [2, Lemma 4.1], it follows that $F$ is coatomic. Furthermore, $F \cap \operatorname{Rad}(M)=\operatorname{Rad}(F)$ by $[4,3.7(3)]$.

(ii) $\Rightarrow$ (viii) Note that $F \cap \Delta_{F}(M)=F \cap \operatorname{Rad}_{F}(M) \cap \Delta_{F}(M)=F \cap \operatorname{Rad}(M)$. Then $F \cap \Delta_{F}(M)=\operatorname{Rad}(F)$ by (ii).

(viii) $\Rightarrow$ (iii) Since $F$ is coatomic, we have $\operatorname{Rad}(F) \ll F$. Thus $F \cap \Delta_{F}(M) \ll F$. But $F \cap \operatorname{Rad}(M) \subseteq F \cap \Delta_{F}(M)$. So $F \cap \operatorname{Rad}(M) \ll F$.

(iii) $\Rightarrow$ (vi) This follows from the fact that $F+\operatorname{Rad}(M)=\operatorname{Rad}_{F}(M)$.

(vi) $\Rightarrow$ (i) Note that $F+\Delta_{F}(M)=M$. In addition, we have $F \cap \Delta_{F}(M) \subseteq$ $F \cap \operatorname{Rad}_{F}(M) \cap \Delta_{F}(M) \subseteq F \cap \operatorname{Rad}(M) \ll F$ by (vi). Therefore $F$ is a supplement of $\Delta_{F}(M)$ in $M$.

The next example shows that the conditions in the hypothesis of Theorem 3.3 are not superfluous.

Example 3.4. (i) Let $p$ be a prime integer and consider the $\mathbb{Z}$-module $M=M_{1} \oplus M_{2}$ where $M_{1}=\mathbb{Z} / p^{2} \mathbb{Z} \oplus 0$ is a maximal submodule of $M$ and $M_{2}=0 \oplus \mathbb{Z} / p \mathbb{Z}$ is simple. It is clear that $M$ is a coatomic module. However, the module $M$ does not have the complete max-property as $M / \operatorname{Rad}(M) \cong \mathbb{Z} / p \mathbb{Z} \oplus \mathbb{Z} / p \mathbb{Z}$ (see [6, Theorems 2.3 and 6.8] or [6, Corollary 6.11]). Let $N=(\overline{1}, \tilde{1}) \mathbb{Z} \leq M$. It is easily seen that $N \oplus M_{2}=M$. So $N$ is a maximal submodule of $M$. Note that $M_{2}$ is a supplement 
in $M$. Moreover, $M_{2} \nsubseteq M_{1}$ and $M_{2} \nsubseteq N$. Hence $\Delta_{M_{2}}(M) \subseteq M_{1} \cap N \subseteq p \mathbb{Z} / p^{2} \mathbb{Z} \oplus 0$. Thus $M_{2}+\Delta_{M_{2}}(M) \subseteq\left(p \mathbb{Z} / p^{2} \mathbb{Z} \oplus 0\right) \oplus M_{2}$. It follows that $M_{2}+\Delta_{M_{2}}(M) \neq M$. This implies that $M_{2}$ is not a supplement of $\Delta_{M_{2}}(M)$ in $M$.

(ii) Let $M$ be a nonzero module with $\operatorname{Rad}(M)=M$. Then $M$ is a supplement in $M$, but $M=M \cap \operatorname{Rad}(M)$ is not small in $M$. Note that $M$ has the complete max-property but $M$ is not coatomic.

Following [2], a module $M$ is called an ms-module if every maximal submodule of $M$ is a supplement in $M$. As an application of Theorem 3.3, we get the following corollaries.

Corollary 3.5. Let $M$ be a coatomic module which has the complete max-property. Then $M$ is an ms-module if and only if $\operatorname{Rad}(M) \ll K$ for every maximal submodule $K$ of $M$.

Corollary 3.6. Let $M$ be a coatomic module which has the complete max-property. Let $L$ and $F$ be submodules of $M$ such that $F \subseteq L$ and $F \cap \operatorname{Rad}(M)=L \cap \operatorname{Rad}(M)$. If $F$ is a supplement in $M$, then so is $L$.

Corollary 3.7. Let $M$ be a coatomic module which has the complete max-property. Let $L$ and $F$ be submodules of $M$ such that $\operatorname{Rad}(M) \subseteq F \subseteq L$. If $F$ is a supplement in $M$, then so is $L$.

Corollary 3.8. Let $M$ be a coatomic module which has the complete max-property and let $N$ be a maximal submodule of $M$. If $N$ and $\Delta_{N}(M)$ are supplements in $M$, then $M$ is an ms-module.

Proof. Let $K$ be a maximal submodule of $M$ such that $K \neq N$. Then $\operatorname{Rad}(M) \subseteq$ $\Delta_{N}(M) \subseteq K$. By Corollary 3.7, it follows that $K$ is a supplement in $M$. Since $N$ is a supplement in $M, M$ is an ms-module.

Corollary 3.9. Let $R$ be a right noetherian ring and let $M$ be a finitely generated $R$-module which has the complete max-property. Then the following statements about a submodule $F$ of $M$ are equivalent:

(i) $F$ is a supplement in $M$;

(ii) $F \cap \operatorname{Rad}(M)=\operatorname{Rad}(F)$.

Proof. Since $R$ is right noetherian and $M$ is finitely generated, every submodule of $M$ is finitely generated. So every submodule of $M$ is coatomic. The result follows from Theorem 3.3.

It is shown in [8, Lemma 1.1] that over a commutative noetherian ring, every submodule of a coatomic module is coatomic. Combining this fact and Theorem 3.3 , we obtain the following result. 
Corollary 3.10. Let $R$ be a commutative noetherian ring and let $M$ be a coatomic $R$-module which has the complete max-property. Then the following statements about a submodule $F$ of $M$ are equivalent:

(i) $F$ is a supplement in $M$;

(ii) $F \cap \operatorname{Rad}(M)=\operatorname{Rad}(F)$.

As noted in [6, p. 80], the class of modules which have the complete maxproperty is not closed under submodules. For example, the $\mathbb{Z}$-module $\mathbb{Q}_{\mathbb{Z}}$ has the complete max-property, however the submodule $\mathbb{Z}$ does not have the complete maxproperty. Next, we will show that for a coatomic module $M$, if $M$ has the complete max-property, then any supplement submodule in $M$ inherits the property.

Proposition 3.11. Let $M$ be a coatomic module. If $M$ has the complete maxproperty, then every supplement submodule of $M$ has the complete max-property.

Proof. Assume that the module $M$ has the complete max-property. Then $M$ is a good module by Proposition 2.10. Let $F$ be a supplement submodule in $M$. Then $M / \Delta_{F}(M)$ has the complete max-property by [6, Lemma 3.4]. Moreover, from Corollary 2.11 and Theorem 3.3, it follows that

$$
F / \operatorname{Rad}(F)=F / F \cap \Delta_{F}(M) \cong\left(F+\Delta_{F}(M)\right) / \Delta_{F}(M)=M / \Delta_{F}(M) .
$$

So $F / \operatorname{Rad}(F)$ has the complete max-property. Using again [6, Lemma 3.4], it follows that $F$ has the complete max-property.

Proposition 3.12. Let $M$ be a module. Assume that $\operatorname{Rad}(M)$ has a supplement $F$ in $M$ such that $F$ has the complete max-property. Then $M$ has the complete max-property.

Proof. By hypothesis, we have $\operatorname{Rad}(M)+F=M$. Then

$$
M / \operatorname{Rad}(M)=(\operatorname{Rad}(M)+F) / \operatorname{Rad}(M) \cong F /(F \cap \operatorname{Rad}(M)) .
$$

Since $F$ has the complete max-property, $F /(F \cap \operatorname{Rad}(M))$ has also the complete max-property by [6, Lemma 3.4]. Therefore $M / \operatorname{Rad}(M)$ has the complete maxproperty. Again by [6, Lemma 3.4], it follows that $M$ has the complete maxproperty.

Proposition 3.13. Let $M=M_{1}+M_{2}$ be a good module such that every maximal submodule of $M$ contains $M_{1}$ or $M_{2}$. Assume that $M_{1}$ and $M_{2}$ are mutual supplements in $M$ and they both have the complete max-property. Then $M$ has the complete max-property. 
Proof. Let $N$ be a maximal submodule of $M$. Without loss of generality we can assume that $M_{1} \subseteq N$. Since $M_{2}$ is a supplement of $M_{1}$, the maximal submodules of $M_{2}$ are $\left\{N_{i} \cap M_{2} \mid i \in I\right\}$ where $\left\{N_{i} \mid i \in I\right\}$ are the maximal submodules of $M$ containing $M_{1}$ by [5, Theorem 2.1]. So $N=N_{i_{0}}$ for some $i_{0} \in I$. Since $M_{2}$ has the complete max-property, we have

$$
\left(N_{i_{0}} \cap M_{2}\right)+\bigcap_{i \neq i_{0}}\left(N_{i} \cap M_{2}\right)=M_{2} .
$$

Let $\left\{N_{j} \mid j \in J\right\}$ be the set of the maximal submodules of $M$ containing $M_{2}$. Hence

$$
N_{i_{0}}+\Delta_{N_{i_{0}}}(M)=N_{i_{0}}+\left(\bigcap_{i \neq i_{0}} N_{i}\right) \bigcap\left(\bigcap_{j \in J} N_{j}\right) .
$$

Since $M$ is a good module, from Theorem 2.3 we have

$$
\bigcap_{j \in J} N_{j}=\operatorname{Rad}_{M_{2}}(M)=M_{2}+\operatorname{Rad}(M) .
$$

Thus,

$$
N_{i_{0}}+\Delta_{N_{i_{0}}}(M)=N_{i_{0}}+\left(\bigcap_{i \neq i_{0}} N_{i}\right) \bigcap\left(M_{2}+\operatorname{Rad}(M)\right) .
$$

By modularity, we get

$$
N_{i_{0}}+\Delta_{N_{i_{0}}}(M)=N_{i_{0}}+\operatorname{Rad}(M)+\left(\left(\bigcap_{i \neq i_{0}} N_{i}\right) \bigcap M_{2}\right) .
$$

But $\operatorname{Rad}(M) \subseteq N_{i_{0}}$. Then, by using $(*)$, we have

$$
\begin{aligned}
N_{i_{0}}+\Delta_{N_{i_{0}}}(M) & =N_{i_{0}}+\bigcap_{i \neq i_{0}}\left(N_{i} \cap M_{2}\right) \\
& =N_{i_{0}}+\left(N_{i_{0}} \cap M_{2}\right)+\bigcap_{i \neq i_{0}}\left(N_{i} \cap M_{2}\right) \\
& =N_{i_{0}}+M_{2} \\
& =M .
\end{aligned}
$$

This completes the proof.

The next example illustrates that the assumption "every maximal submodule of $M$ contains $M_{1}$ or $M_{2}$ "in Proposition 3.13 cannot be dropped.

Example 3.14. Let $M$ be as in Example 3.4(i). The module $M$ does not have the complete max-property. Since $M / \operatorname{Rad}(M)$ is semisimple, $M$ is a good module. Also, $M_{1}$ and $M_{2}$ are mutual supplements in $M$. Let $N=(\overline{1}, \tilde{1}) \mathbb{Z} \leq M$. It is easily seen that $N$ is a maximal submodule of $M$ such that neither $M_{1}$ nor $M_{2}$ is 
contained in $N$. Note that both of $M_{1}$ and $M_{2}$ have the complete max-property since each one of them has only one maximal submodule.

Combining Proposition 3.13 and [6, Lemma 3.4], we obtain the following result.

Corollary 3.15. Let $M=M_{1} \oplus M_{2}$ be a good module such that every maximal submodule of $M$ contains $M_{1}$ or $M_{2}$. Then $M$ has the complete max-property if and only if $M_{1}$ and $M_{2}$ have the complete max-property.

In the next result, we evaluate $\Delta_{F}(M)$ for a supplement submodule $F$ of a coatomic module $M$ which has the complete max-property.

Theorem 3.16. Let $M$ be a coatomic module which has the complete max-property and let $K$ be a submodule of $M$. Let $F$ be a supplement of $K$ in $M$. Then

$$
\Delta_{F}(M)=K+\operatorname{Rad}(F)=K+\operatorname{Rad}(M) .
$$

Proof. Set $\Gamma=\{L \leq M \mid L$ is maximal in $M$ and $F \nsubseteq L\}$ and $\Lambda=\{N \leq M \mid$ $N$ is maximal in $M$ and $K \subseteq N\}$. Clearly $\Lambda \subseteq \Gamma$. Let us show that $\Lambda=\Gamma$. Note that $F$ is a supplement of $\Delta_{F}(M)$ in $M$ by Theorem 3.3. It follows that for a maximal submodule $X$ of $M, F \nsubseteq X$ if and only if $\Delta_{F}(M) \subseteq X$. Let $L \in \Gamma$. Then $\Delta_{F}(M) \subseteq L$. By [5, Proof of Theorem 2.1], $L \cap F$ is a maximal submodule of $F$ and $N=(L \cap F)+K$ is a maximal submodule of $M$. Note that $N \cap F=((L \cap F)+K) \cap F=(L \cap F)+(K \cap F)$. As $F$ is a supplement of $K$ in $M$, we have $K \cap F \ll F$. So $K \cap F \subseteq \operatorname{Rad}(M) \subseteq L$. Thus $K \cap F \subseteq L \cap F$. Hence $N \cap F=L \cap F$. Note that $F \nsubseteq N$. Then $\Delta_{F}(M) \subseteq N$. By modularity, we have

$L=L \cap\left(F+\Delta_{F}(M)\right)=(L \cap F)+\Delta_{F}(M)=(N \cap F)+\Delta_{F}(M)=N \cap\left(F+\Delta_{F}(M)\right)=N$.

It follows that $L \in \Lambda$. So $\Lambda=\Gamma$. Thus $\Delta_{F}(M)=\operatorname{Rad}_{K}(M)$. Since $M$ is good, $\Delta_{F}(M)=\operatorname{Rad}_{K}(M)=K+\operatorname{Rad}(M)$ by Theorem 2.3. Moreover, by Theorem 3.3, we have $F \cap \Delta_{F}(M)=\operatorname{Rad}(F)$. So $\Delta_{F}(M)=(K+F) \cap \Delta_{F}(M)=K+(F \cap$ $\left.\Delta_{F}(M)\right)=K+\operatorname{Rad}(F)$.

Remark 3.17. Let $M$ be a coatomic module which has the complete max-property and let $F$ be a supplement in $M$. From the previous result, it follows that if $F$ is a supplement of a submodule $K$ in $M$, then

(i) $K \subseteq \Delta_{F}(M)$, and

(ii) every maximal submodule of $M$ contains $F$ or $K$.

By the following example we see that the condition " $M$ has the complete maxproperty" cannot be omitted from the hypothesis of Theorem 3.16. 
Example 3.18. Let $M$ be as in Example 3.4(i). So $M_{2}$ is a supplement of both $M_{1}$ and $N$ in $M$. Since $M_{1}$ and $N$ are maximal submodules of $M$, we have $N+$ $\operatorname{Rad}(M)=N$ and $M_{1}+\operatorname{Rad}(M)=M_{1}$. Thus $N+\operatorname{Rad}(M) \neq M_{1}+\operatorname{Rad}(M)$. Note that $M$ is a coatomic module which does not have the complete max-property.

As an application of Theorem 3.16, we obtain the following two propositions.

Recall that following [1], two submodules $X$ and $Y$ of a module $M$ are said to be $\beta^{*}$ equivalent (denoted as $X \beta^{*} Y$ ) if $(X+Y) / X \ll M / X$ and $(X+Y) / Y \ll M / Y$. It was shown in [1, Theorem 2.6 (ii)] that if $X, Y$ are submodules of $M$ such that $X \beta^{*} Y$, then $X$ has a supplement $C$ in $M$ if and only if $C$ is a supplement of $Y$ in $M$.

Proposition 3.19. Let $M$ be a coatomic module which has the complete maxproperty and let $H, K$ and $F$ be submodules of $M$. Assume that $F$ is a supplement of both $H$ and $K$ in $M$. Then $H \beta^{*} K$.

Proof. By Theorem 3.16, we have $H+\operatorname{Rad}(M)=K+\operatorname{Rad}(M)=\Delta_{F}(M)$. From [1, Corollary 2.4], it follows that $H \beta^{*} K$.

Following [1], a module $M$ is called Goldie*-supplemented if for every submodule $X$ of $M$, there exists a supplement submodule $F$ in $M$ such that $X \beta^{*} F$. It was shown in [1, Theorem 3.6 and Example 3.9 (iii)] that any Goldie*-supplemented module is supplemented but the converse is not true, in general. In the next proposition, we present some sufficient conditions for a supplemented module to be Goldie*-supplemented.

Proposition 3.20. Let $M$ be a coatomic module which has the complete maxproperty. If $M$ is supplemented, then $M$ is Goldie*-supplemented

Proof. Assume that $M$ is a supplemented module. Let $X$ be a submodule of $M$. Let $F$ be a supplement of $X$ in $M$ and let $T$ be a supplement of $F$ in $M$. Then $F$ is a supplement of $T$ in $M$ by [4, 20.4 (9)]. Using Theorem 3.16, we get $X+\operatorname{Rad}(M)=T+\operatorname{Rad}(M)=\Delta_{F}(M)$. Note that $\operatorname{Rad}(M) \ll M$. Therefore $M$ is Goldie*-supplemented by [1, Corollary 3.4].

Acknowledgement. The authors would like to thank the referee for the valuable suggestions and comments. 


\section{References}

[1] G. F. Birkenmeier, F. Takil Mutlu, C. Nebiyev, N. Sokmez and A. Tercan, Goldie*-supplemented modules, Glasg. Math. J., 52(A) (2010), 41-52.

[2] E. Büyükasik and D. Pusat-Yilmaz, Modules whose maximal submodules are supplements, Hacet. J. Math. Stat., 39(4) (2010), 477-487.

[3] M. D. Cissé and D. Sow, On generalizations of essential and small submodules, Southeast Asian Bull. Math., 41(3) (2017), 369-383.

[4] J. Clark, C. Lomp, N. Vanaja and R. Wisbauer, Lifting Modules, Supplements and Projectivity in Module Theory, Frontiers in Mathematics, Birkhäuser Verlag, Basel, 2006.

[5] C. Nebiyev and A. Pancar, On supplement submodules, Ukrainian Math. J., 65(7) (2013), 1071-1078.

[6] P. F. Smith, Module with coindependent maximal submodules, J. Algebra Appl., 10(1) (2011), 73-99.

[7] R. Wisbauer, Foundations of Module and Ring Theory, Algebra, Logic and Applications, 3, Gordon and Breach Science Publishers, Philadelphia, PA, 1991.

[8] H. Zöschinger, Koatomare moduln, Math. Z., 170(3) (1980), 221-232.

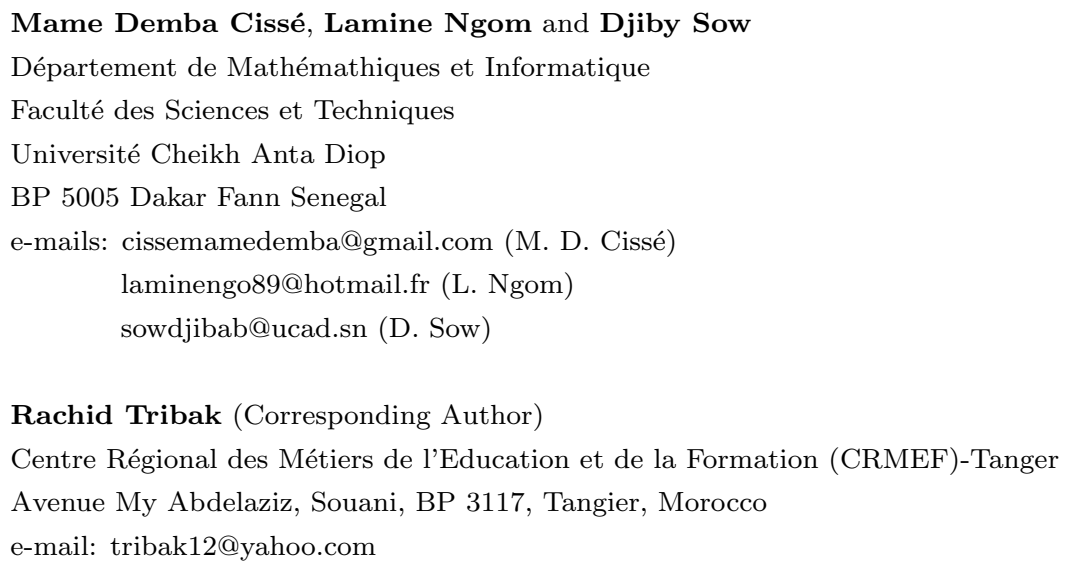

